\title{
LA SUBLUXACIÓN ROTATORIA DE COLUMNA EN LA NEUROFIBROMATOSIS TIPO I: APLICACIÓN DE LA VÍA INFRAAXILAR.
}

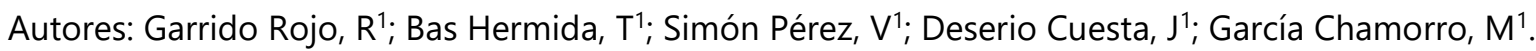

${ }^{1}$ HospitalUniveristari I Politècnic La Fe,Valencia, Spain

Resumen

Introducción

El $60 \%$ de los pacientes con neurofibromatosis asocian distrofias vertebrales y deformidades de la columna, la mayoría cifoescoliosis altas y muy angulosas, donde las vértebras progresivamente se dislocan ejerciendo un cizallamiento del canal medular que da lugar a severas alteraciones neurológicas. En estos pacientes continúa siendo un reto el abordaje de la deformidad mediante las vías habituales; por ello describimos una reciente técnica realizando una toracotomía infraaxilar.

\section{Material y métodos}

Presentamos el caso de una mujer de 9 años con NF tipo I y cifoescoliosis dorsal alta de convexidad izquierda (doble curva

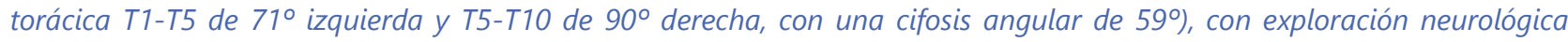
normal. Previo periodo de tracción raquídea nos disponemos a realizar una artrodesis posterior, pero al colocar a la paciente en decúbito prono en la mesa quirúrgica se produce una abolición de los potenciales evocados motores que obliga a suspender la intervención.

\section{Resultados}

La solución fue realizar una toracotomía infraaxilar izquierda, abordando la curva desde la convexidad. Se colocó a la paciente en decúbito lateral derecho a $70^{\circ}$ y se realizó una resección costal dos o tres niveles por encima del ápex de la deformidad. Después de una cuidadosa disección manteniéndose cerca de la pared torácica, se realizó una descompresión y discetomía anterior a nivel del ápex. A continuación se colocó en la concavidad dos aloinjertos tibiales "en puntal», a modo de empalizada, para soportar las fuerzas de compresión. En un segundo tiempo se realizó un abordaje posterior instrumentado para conseguir una artrodesis circunferencial y una óptima estabilidad.

\section{Conclusión}

La técnica presentada permite el acceso a la columna torácica alta de forma directa y segura, al no implicar estructuras vitales incluyendo los grandes vasos o el conducto torácico como lo podían hacer los abordajes clásicos. Se trata de una técnica prometedora con buenos resultados clínicos, que puede ser utilizada para el tratamiento de las deformidades cifóticas donde se ha visto que los abordajes posteriores únicos fallan, pero también de inestabilidades, compresiones medulares u otras patologías del cuerpo vertebral ya que puede conseguir una buena exposición de los mismos.

PALABRAS CLAVE: "cifoescoliosis", "neurofibromatosis", "abordaje infraaxilar", "subluxación rotatoria".

Autor de correspondencia:

Garrido Rojo R. Mail: raquelgr3293@gmail.com

Hospital Universitario y Politécnico la Fe.

Avda Fernando Abril Martorell 106, Valencia, España. 


\section{INTRODUCCIÓN}

La neurofibromatosis es una enfermedad hereditaria que afecta a los tejidos derivados de las tres estirpes embrionarias: el neuroectodermo, el endodermo y el mesodermo. Por tanto, las manifestaciones clínicas son muy variables, y pueden afectar a la piel, al sistema nervioso, al esqueleto y a los tejidos blandos. Existen dos tipos de neurofibromatosis. El tipol es el más frecuente y los criterios para establecer su diagnóstico son la presencia de manchas cutáneas de «café con leche», neurofibromas, gliomas del nervio óptico, hamartomas del iris y anomalías del sistema musculoesquelético ${ }^{[1]}$. En conjunto, el $60 \%$ de los pacientes con neurofibromatosis de tipo I tienen deformidades de la columna vertebral, que pueden asociarse o no a una distrofia ósea de las vértebras. Este componente distrófico puede ir acompañado de anomalías del canal medular secundarias a una malformación medular. Las deformidades más comunes son las escoliosis, que afectan al $2-36 \%$ de los pacientes portadores de una neurofibromatosis tipo I (NF1). Casi todas ellas son cortas y angulosas y aparecen en etapas precoces de la infancia. Las cifosis puras son raras, y las cifoescoliosis mucho más comunes ${ }^{[2]}$.

En las cifoescoliosisdorsales hay un neto predominio de la cifosis con respecto a la deformidad puramente escoliótica. Los cuerpos vertebrales están a menudo tan deformados que es imposible analizarlos en las radiografías habituales.

EI TC permite un estudio completo de cada vértebra distrófica y de los contornos del canal raquídeo. Las reconstrucciones tridimensionales proporcionan un análisis morfológico detallado en los tres planos del espacio. Con la RM se puede estudiar la médula y visualizar las zonas de afectación medular o las posibles malformaciones medulares asociadas. También puede detectarse la presencia de ectasias de la duramadre, que a veces producen una erosión progresiva de los cuerpos vertebrales y desestabilización de la columna (fenómeno de "scalloping» $u$ «ondulación») $)^{[2]}$.

Subluxación rotatoria vertebral

El término "subluxación rotatoria" fue acuñado y descrito por Duval-Beaupere y Dubousset en 1972, en un estudio donde analizaron 11 pacientes con algún tipo de distrofia vertebral debido a neurofibromatosis $y$ otras displasias congénitas $^{[3]}$.

Vieron que en este tipo de enfermedades las dos vértebras distróficas localizadas en la unión de dos segmentos de curvas escolióticas (cada una de ellas lordótica y rotada en direcciones opuestas) progresivamente se dislocan o subluxan adoptando una configuración cifótica y rotacional, ejerciendo un efecto de cizalla alrededor del canal medular. En las radiografías AP y lateral se demuestra que el ápex de la cifosis está localizado exactamente en la unión de las dos curvas escolióticas vistas en la AP.

Este fenómeno va progresando con el tiempo. La «horquilla» que se forma es la responsable de una deformidad en bayoneta del canal raquídeo. Esta deformidad progresiva es, a su vez, responsable del estiramiento de la médula en el canal raquídeo $\mathrm{y}$, por tanto, el posible origen de alteraciones neurológicas.

Estas deformidades producen también insuficiencia respiratoria por alteración de la forma e incompetencia de la jaula torácica.

Tratamiento precoz: artrodesis
circunferencial

Es importante ser conscientes de que en las formas distróficas de cifoescoliosis como en la NF cabe la posibilidad de que se produzca con el tiempo una subluxación rotacional progresiva que produzca 
complicaciones neurológicas como paraplejías progresivas, por lo que la estabilización debe ser precoz ${ }^{[3,4,5]}$.

A menudo se precisa un período previo de tracción raquídea para obtener una corrección máxima y progresiva, con posible recuperación de las alteraciones neurológicas y mejor preparación respiratoria. Las intervenciones de fusión instrumentada por vía posterior aislada suelen estar condenadas al fracaso, con recidiva de la deformidad ${ }^{[6,7]}$.

Hay que lograr un apoyo anterior sólido, por lo general con ayuda de injertos peroneos autólogos "en puntal" ${ }^{2,3]}$. Resulta preferible comenzar por el tiempo anterior, con el fin de no correr el riesgo de desestabilizar por completo la columna como consecuencia de las posibles extirpaciones óseas hechas con anterioridad por vía posterior, y para mantener la corrección que se obtuvo con la tracción preoperatoria.

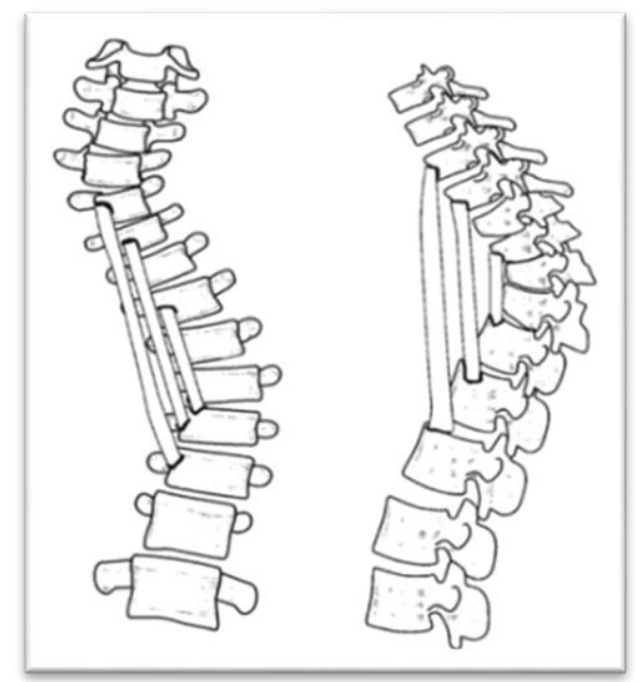

Después de un tiempo se lleva a cabo la artrodesis posterior. La corrección de la deformidad espinal en este punto representa una maniobra de descompresión del canal medular que requiere una torsión severa del ápex de la cifosis, por lo que se debe realizar con un especial cuidado.
La inmovilización, casi siempre mediante un sistema de corsé acoplado a un halo craneal, debe ser prolongada, con una duración total de 6 meses a 1 año.

Una nueva vía de abordaje: vía infraaxilar Los trastornos de la columna torácica alta pueden conducir a severas complicaciones y morbilidades, y sin embargo operar sobre ella continúa siendo un reto debido a las características de la anatomía a este nivel.

Recientemente se ha descrito una nueva técnica de abordaje de la columna torácica alta que hasta el momento ha resultado ser segura y fácilmente reproducible, la toracotomía infaaxilar ${ }^{[8]}$, una vía utilizada normalmente en cirugía cardíaca y torácica. Esta vía de abordaje nos permite el acceso directo a los cuerpos vertebrales desde T2 a T6 inclusive, evitando el riesgo de dañar estructuras anatómicas complejas que estaban en peligro en las vías de abordaje anterior clásicas, como son la vía transesternal o transtorácica alta, donde encontramos estructuras vitales como el conducto torácico y los grandes vasos.

Mediante esta vía torácica anterolateral podemos exponer de forma segura los cuerpos vertebrales de la columna torácica alta y tratar así patologías del cuerpo vertebral que pueden causar compresiones medulares, inestabilidades espinales 0 deformidades cifóticas, por enfermedades como tumores metastásicos, abscesos fríos o deformidades progresivas como es el caso de este artículo. Todo ello evitando el mediastino y por tanto minimizando el riesgo de dañar estructuras como el esófago, la pleura, el nervio laríngeo recurrente, el nervio vago o los grandes vasos.

\section{MATERIAL Y MÉTODOS}

En el presente artículo presentamos el caso de una mujer de 9 años con neurofibromatosis tipo I, que presentaba 
una cifoescoliosis dorsal alta de convexidad izquierda: doble curva torácica T1-T5 de $71^{\circ}$ izquierda y T5-T10 de $90^{\circ}$ derecha, con una cifosis angular de $59^{\circ}$. Sin malformaciones óseas en TC y sin compromiso medular en $\mathrm{RM}$, apreciándose en esta última un pinzamiento de los discos afectados por el segmento de la escoliosis.

A la exploración física presentaba:

- Múltiples manchas café con leche.

- Área de rotación fija torácica izquierda.

- Hombro izquierdo ligeramente ascendido.

- Pelvis derecha ligeramente ascendida.

- Borramiento pliegue izquierdo.

- Contractura de isquiotibiales.

La exploración neurológica periférica estaba conservada y presentaba un patrón de restricción respiratoria en las pruebas funcionales.
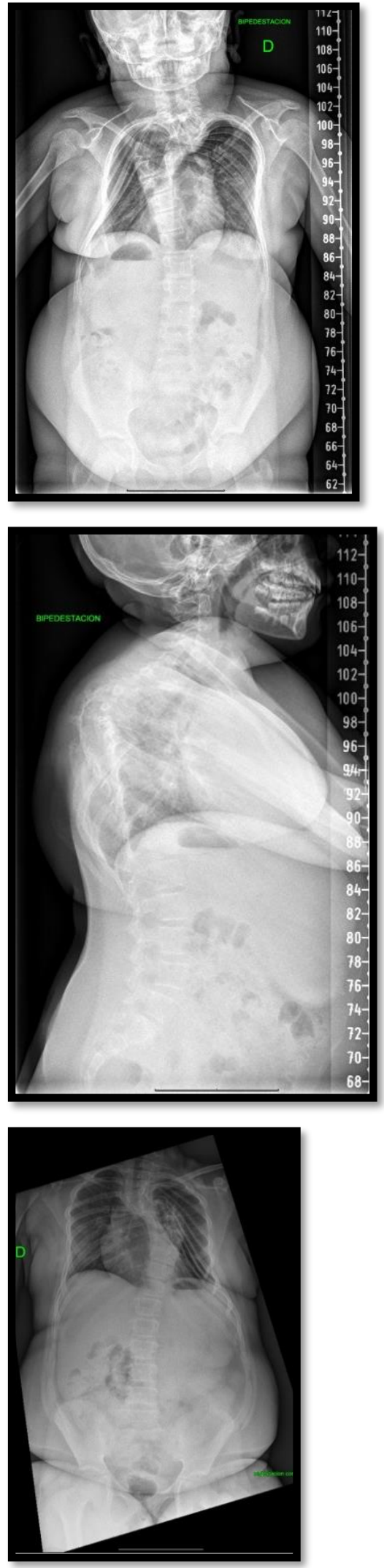

Imagen 1: Radiografías de la paciente previas a la intervención, durante el ingreso con halo-tracción. 


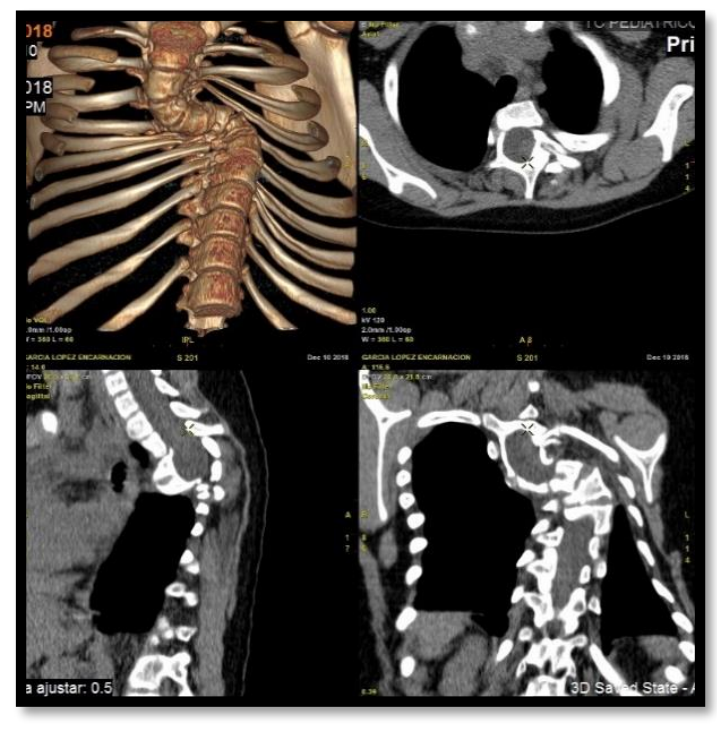

Imagen 2: TC y reconstrucciones en 3D

En primer lugar, se llevó a cabo un período de un mes de tracción raquídea con halo craneal, para lograr un estiramiento progresivo de la cifosis y obtener así la máxima corrección posible. Durante este período la exploración neurológica diaria fue normal.

Tras esta preparación nos disponemos a realizar una artrodesis posterior instrumentada para el tratamiento de la deformidad, con la sorpresa de que al colocar a la paciente en decúbito prono se produce una abolición de los potenciales motores y sensitivos en miembros inferiores que obliga a suspender la intervención.
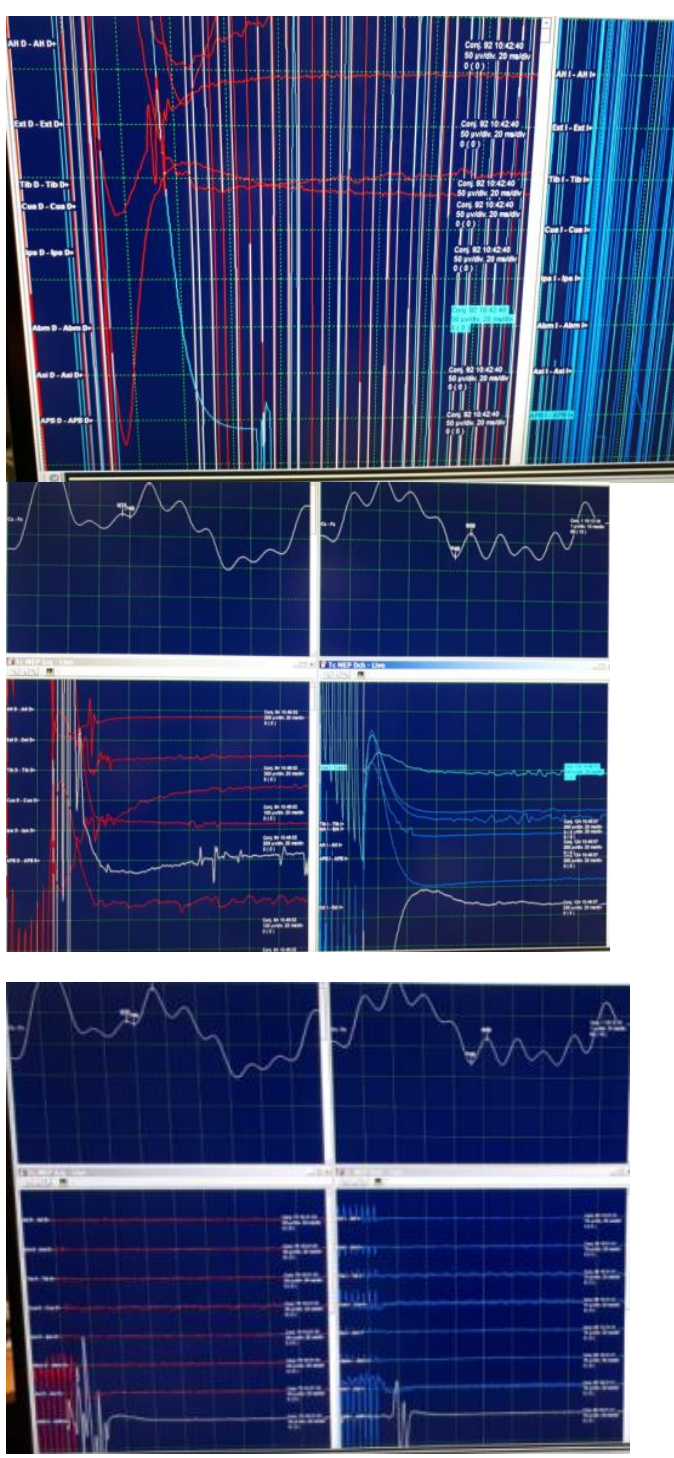

Imagen 3: imágenes intraoperatorias de la monitorización neurofisiológica donde se aprecia la pérdida de PESS y PEM en MMII durante la inducción anestésica.

La conducción fue recuperada con el decúbito supino. Tras esta eventualidad, se realizó una actualización de todos los estudios y una ampliación de los mismos con RM, angio-TC y reconstrucciones en 3D. 


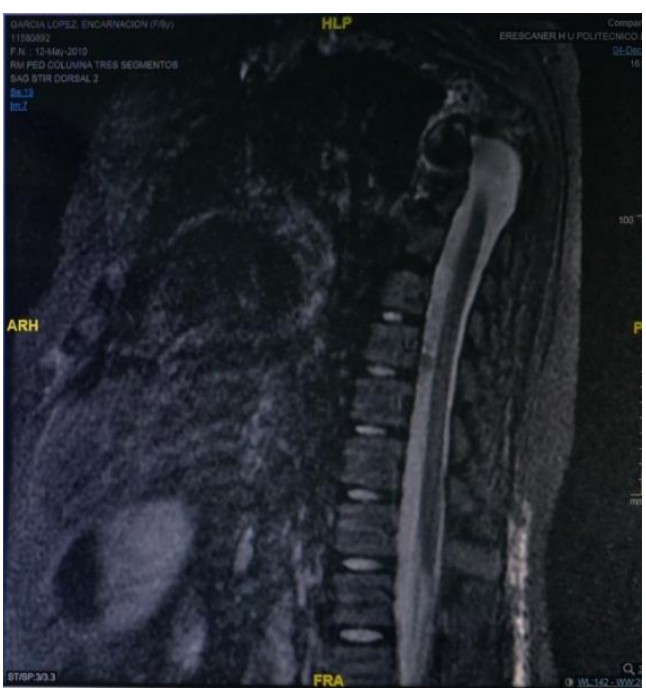

Imagen 4: Corte de RM donde se muestra un importante acodamiento de la médula en el ápex de la deformidad a nivel T4-T6, sin identificarse cambios de señal intramedular sugestivos de mielopatía ni anomalías vertebrales.

\section{RESULTADOS}

Ante la sospecha de que pudiera tratarse de una subluxación rotatoria de la columna torácica alta, se decidió que el tratamiento más adecuado sería realizar una artrodesis circunferencial con una doble vía de abordaje, comenzando por una vía anterior en un primer tiempo.

En la técnica original se describe el abordaje anterior desde la concavidad de la curva escoliótica inferior. Sin embargo, en nuestro caso decidimos abordar la curva por su parte convexa, ya que por la anatomía de la misma la curva queda mejor expuesta y se evita la manipulación de los grandes vasos.

Se colocó a la paciente en decúbito lateral derecho. Se realizó una toracotomía anterolateral izquierda a nivel infraaxilar, con resección costal dos o tres niveles por encima del ápex de la deformidad.

Después de una cuidadosa disección manteniéndose cerca de la pared torácica, el ápex de la deformidad queda al descubierto. Para ganar acceso al ápex, los vasos segmentarios relacionados con estas vértebras fueron ligados. Se realizó una descompresión y discetomía anterior a nivel del ápex.

A continuación, se obtuvo un injerto de tibia de cadáver, de la cual se confeccionaron dos injertos con forma de puntal. En la técnica original se describe la obtención del injerto de la propia tibia o peroné del paciente, incluso el uso de las costillas reseccionadas. En nuestra paciente se desestimó esta opción debido a las posibles características displásicas de ese hueso por su NF de base. Se eligió además tibia por su mayor abundancia en hueso esponjoso.

\section{Estos dos injertos de tibia "en puntal"} fueron insertados en la concavidad de la cifosis a modo de empalizada o arbotante, comenzando por uno corto a nivel del ápex de la cifosis. De este modo, en la concavidad, soportarán las fuerzas de compresión. Debido a la orientación de los cuerpos vertebrales, los injertos fueron alineados con la gravedad en el plano sagital y más o menos oblicuos en el plano coronal. Los huecos existentes entre los injertos tibiales fueron rellenados con chips de hueso esponjoso. 


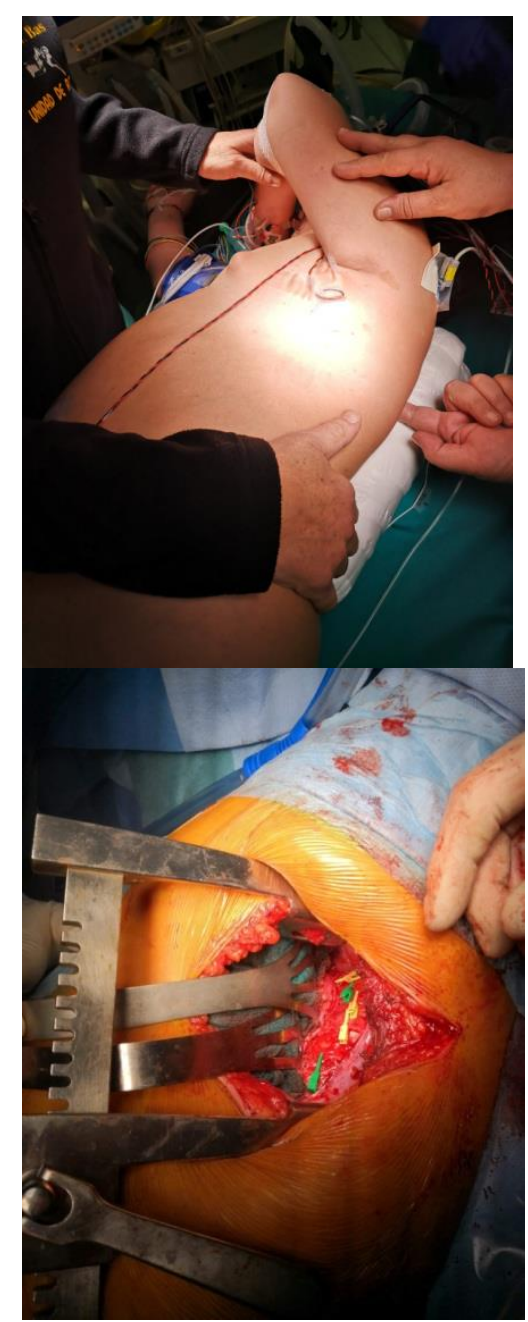

Imagen 5: Fotografías intraoperatorias sobre el posicionamiento de la paciente en decúbito lateral derecho y el abordaje infraaxilar izquierdo realizado.

Un mes después, tras la recuperación de la paciente y su correcta evolución clínica y radiológica, se procede a realizar el segundo tiempo quirúrgico mediante una vía posterior, también con monitorización multimodal intraoperatoria, para completar la artrodesis circunferencial.

En este caso se realizó un abordaje desde C7 a T12, osteotomías tipo Ponte a nivel del ápex de la deformidad e instrumentación posterior con tornillos pediculares y uñas costales. Los potenciales se mantuvieron conservados en todo momento.

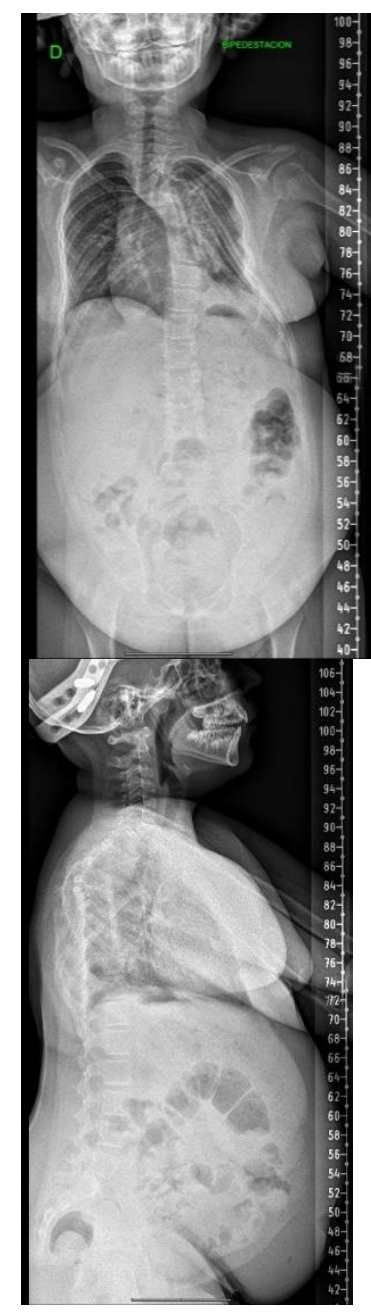

Imagen 6: Radiografías anteroposterior $y$ lateral de columna completa en bipedestación después del primer tiempo post-operatorio.

Al seguimiento un año después, la paciente se encuentra asintomática, realizando vida normal y deporte. La evolución ha sido satisfactoria, sin complicaciones neurológicas hasta el momento. 

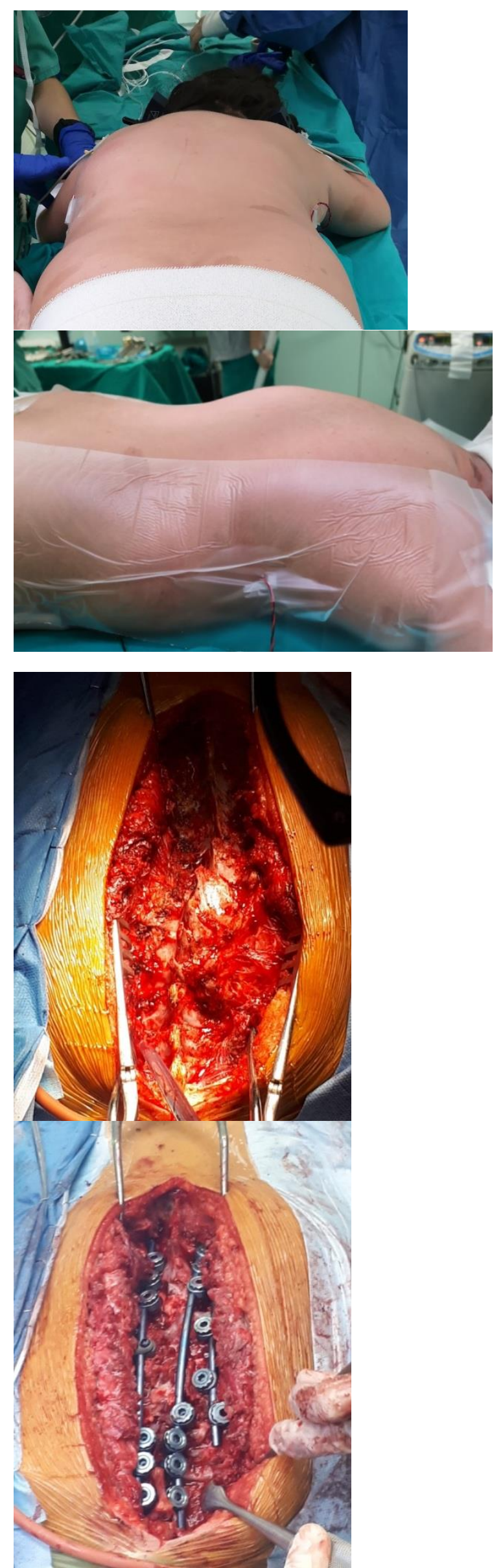

Imagen 7: Fotografías intraoperatorias durante el segundo tiempo quirúrgico con la paciente en decúbito prono, vía de abordaje posterior e instrumentación.
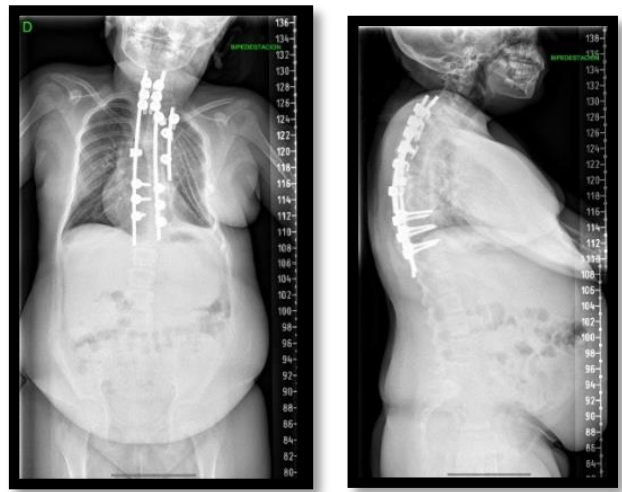

Imagen 8: Radiografías anteroposterior $y$ lateral de columna completa en bipedestación tras el segundo tiempo quirúrgico.

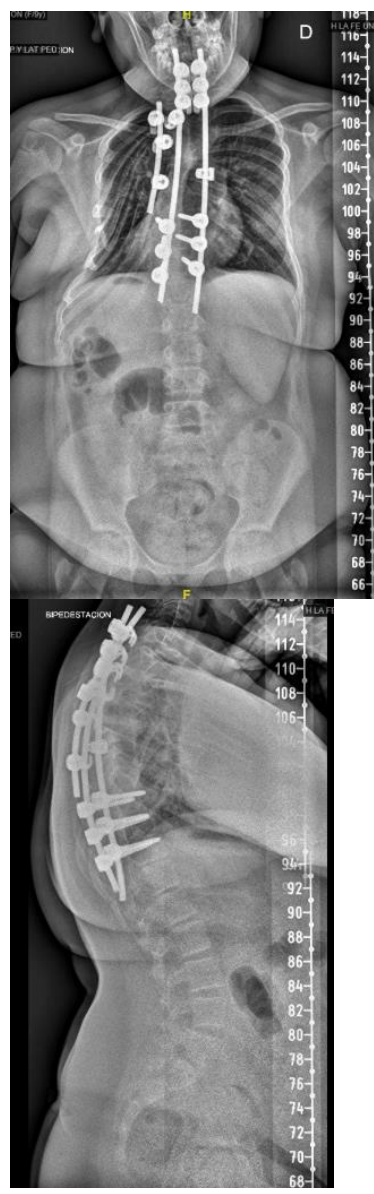




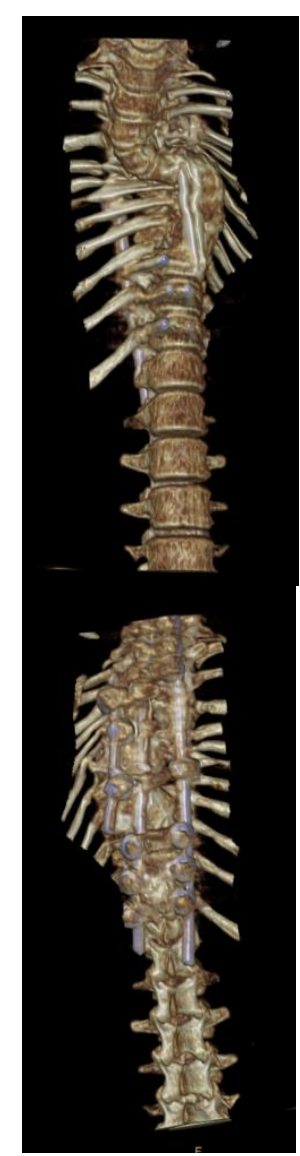

Imagen 9: Radiografías anteroposterior $y$ lateral de columna completa en bipedestación y cortes de reconstrucción de $T C$ en $3 D$ al seguimiento un año después de la intervención.

\section{CONCLUSIONES}

El debut de una subluxación rotatoria dentro de una cifoescoliosis es una de las formas más graves de deformidad de la columna espinal, que puede acarrear graves consecuencias neurológicas.

Su diagnóstico precoz es fundamental, y se basa en la identificación apropiada del patrón de la curva: el ápex de la cifosis en la radiografía lateral se corresponde con la zona de unión de dos curvas escolióticas en la radiografía anteroposterior.

Esta deformidad, la cifosis angular que existe incluso en estadios precoces de la enfermedad, va progresando rápidamente hasta producir déficits neurológicos, por lo que la estabilización precoz es fundamental.

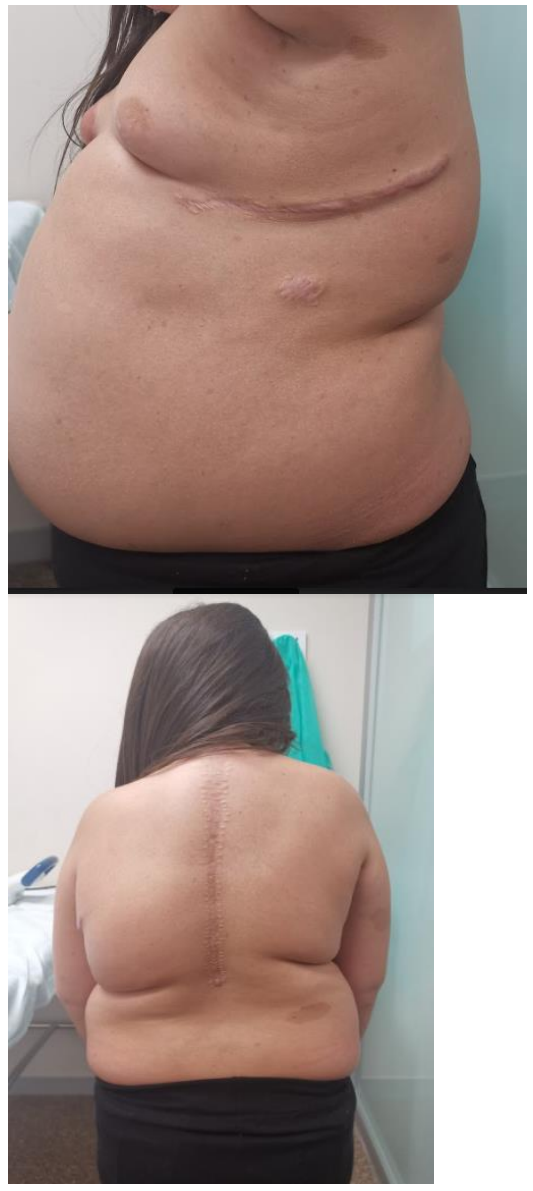

Imagen 10: Fotografías clínicas de la paciente en consulta en el seguimiento al año de la intervención.

Se recomienda realizar una artrodesis circunferencial con injertos en puntal por vía anterior, ya que se ha visto que en la mayoría de ocasiones los abordajes posteriores únicos fallan.

Para la realización de esta vía anterior se recomienda la aplicación del abordaje infraaxilar por toracotomía, una reciente vía de abordaje que según la experiencia hasta el momento es fácilmente reproducible y nos permite tener un acceso directo a los cuerpos vertebrales de la columna torácica alta de una forma segura, con una buena visualización de los mismos y adecuado espacio para operar, minimizando el riesgo de daño de las estructuras vitales presentes adyacentemente. 
*Todas las imágenes se obtuvieron y fueron utilizadas con el consentimiento firmado de ambos progenitores y de acuerdo con la paciente. Se declara que no existen conflictos de intereses.

\section{BIBLIOGRAFÍA}

David H. Gutmann et al (2017). Neurofibromatosis type 1. Nature Reviews Disease Primers volume 3, Article number: 17004. doi: $10.1038 /$ nrdp.2017.4

Garreau de Loubresse, Christian \& Vialle, R \& Wolff, Stéphane. (2005). Cifosis patológicas. EMC - Aparato Locomotor. 38. 1-32. doi:10.1016/S1286-935X(05)43404-8.

Zeller, Reinhard \& Dubousset, Jean (2000). Progressive Rotational Dislocation in Kyphoscoliotic Deformities. Spine, 25, $1092-$ 7. doi:10.1097/00007632-200005010-00009.

Dhokia, Rakesh et al (2017). The Treatment of Rotatory Dislocation of the Spine in a Patient With Dystrophic Kyphoscoliosis Secondary to Neurofibromatosis Type I. The Spine Journal, Volume 17, Issue 11, S333. doi: 10.1016/j.spinee.2017.10.056 - un caso de un buen resultado de la artrodesis circunferencial, señala la necesidad de tratamiento por progresión de la curva después de la maduración ósea.

Tsirikos, A. I., Dhokia, R., \& Wordie, S. (2018). Rotatory Dislocation of the Spine in Dystrophic Kyphoscoliosis Secondary to Neurofibromatosis Type 1. Journal of central nervous system disease, 10, 1179573518819484.

doi:10.1177/1179573518819484

Singh, Kern et al (2005). Neurofibromatosis type I with severe dystrophic kyphoscoliosis and its operative management via a simultaneous anterior-posterior approach: a case report and review of the literatura. The
Spine Journal, Volume 5, Issue 4, 461 466. doi: 10.1016/j.spinee.2004.09.015

Meneses-Quintero D, Alvarado-Gómez F, Alcalá-Cerra G. Dystrophic thoracic spine dislocation associated with type-1 neurofibromatosis: Case report and rationale for treatment. J Craniovert Jun Spine 2015;6:79-82. doi: 10.4103/09748237.156067

Liu, J., Li, S., Huang, K. et al. Right infraaxillary thoracotomy approach for upper thoracic vertebraldecompression and fusion at T2-T6 levels: a technical note. Eur Spine J (2019) 28: 470. https://doi.org/10.1007/s00586-018-5686-x 